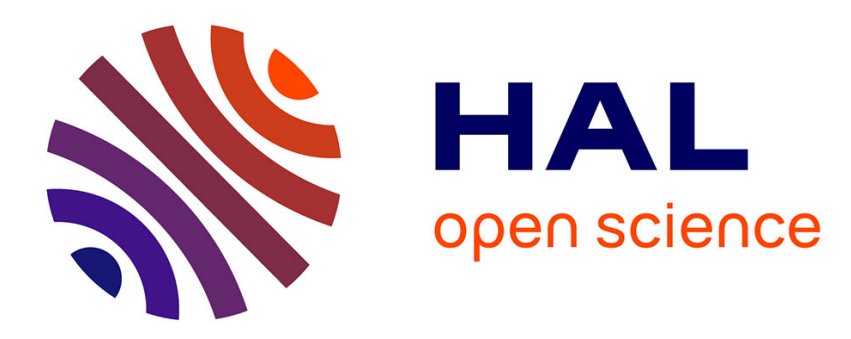

\title{
Determination of two-particle structure factors from elemental maps
}

C. Kreyenschulte, H. Kohl

\section{To cite this version:}

C. Kreyenschulte, H. Kohl. Determination of two-particle structure factors from elemental maps. European Physical Journal: Applied Physics, 2011, 54 (3), 10.1051/epjap/2010100356 . hal-00705150

\section{HAL Id: hal-00705150 \\ https://hal.science/hal-00705150}

Submitted on 7 Jun 2012

HAL is a multi-disciplinary open access archive for the deposit and dissemination of scientific research documents, whether they are published or not. The documents may come from teaching and research institutions in France or abroad, or from public or private research centers.
L'archive ouverte pluridisciplinaire HAL, est destinée au dépôt et à la diffusion de documents scientifiques de niveau recherche, publiés ou non, émanant des établissements d'enseignement et de recherche français ou étrangers, des laboratoires publics ou privés. 
EPJ manuscript No.

(will be inserted by the editor)

\title{
Determination of Two-particle Structure Factors from Elemental Maps
}

\author{
Carsten Kreyenschulte and Helmut Kohl
}

Physikalisches Institut and Interdisziplinäres Centrum für Elektronenmikroskopie und Mikroanalyse (ICEM), University of Münster, Wilhelm-Klemm-Strasse 10, 48149 Münster, Germany

carsten.kreyenschulte@uni-muenster.de

Received: date / Revised version: date

\begin{abstract}
The structure of crystals can be described by defining size and shape of a unit cell and the positions of the atoms within it. Many materials, however, exhibit a glassy or amorphous structure. Such disordered structures are described by structure factors. These are usually determined by small angle scattering experiments. The angular distribution recorded in these experiments is related to the structure factor. In this work we present an alternative approach using elemental maps obtained in an energy filtering transmission electron microscope. In this way we can even obtain chemically resolved partial structure factors giving additional information on the specimen.
\end{abstract}

\section{Introduction}

To understand the properties of a material, a detailed knowledge of the chemical composition of it is essential to understand its properties. Therefore, methods to determine the elemental distribution with high spatial resolution are of great interest in materials science. In a pioneering manuscript, Christian Colliex et al. demonstrated about 40 years ago that inelastically scattered electrons can be used to obtain an element-specific signal. Using a transmission electron microscope, equipped with an imaging energy filter of the Castaing-Henry type, he obtained images showing the distribution of copper and silicon in an $\mathrm{Cu}-\mathrm{Si}$ alloy [1].

Here we demonstrate an application of this technique to disordered structures.

When analysing specimens of disordered solids we have to find a way to describe a random structure and to obtain experimental data from them. One approach is to use small angle neutron scattering (SANS) or small angle x-ray scattering (SAXS) to arrive at the two particle structure factor $[2,3]$. Both methods require fairly large specimen sizes. Also a single scattering experiment is insensitive to the chemical composition for multi-element specimens, which may have element specific partial structure factors. To illustrate this, we consider the differential scattering cross section $d \sigma / d \Omega$ in first order Born approximation giving the angular distribution in a scattering experiment

$$
d \sigma / d \Omega \propto|n(\boldsymbol{u})|^{2}|f(u)|^{2},
$$

where $n(\boldsymbol{u})$ is the Fourier-transformed particle density, $f(u)$ the atomic scattering amplitude and $u$ the spatial frequency [4]. The structure factor $S(u)$ is defined as the rotational average $\langle\ldots\rangle$ of $|n(\boldsymbol{u})|^{2}$. Extending (1) to two elements $a$ and $b$, we obtain

$$
\begin{aligned}
\langle d \sigma / d \Omega\rangle \propto & \left\langle\left|n_{a}(\boldsymbol{u}) f_{a}(u)+n_{b}(\boldsymbol{u}) f_{b}(u)\right|^{2}\right\rangle \\
\propto & \left\langle\left|n_{a}(\boldsymbol{u})\right|^{2}\right\rangle\left|f_{a}(u)\right|^{2}+\left\langle\left|n_{b}(\boldsymbol{u})\right|^{2}\right\rangle\left|f_{b}(u)\right|^{2} \\
& +2 \operatorname{Re}\left\langle n_{a}(\boldsymbol{u}) n_{b}^{*}(\boldsymbol{u})\right\rangle f_{a}(u) f_{b}^{*}(u) \\
\propto & S_{a a}(u)\left|f_{a}(u)\right|^{2}+S_{b b}(u)\left|f_{b}(u)\right|^{2} \\
& +S_{a b}(u) f_{a}(u) f_{b}^{*}(u) .
\end{aligned}
$$

Here we have defined the partial structure factors $S_{a a}(u)$ and $S_{b b}(u)$ for the respective elements and the mixed structure factor $S_{a b}(u)[2]$.

Unfortunately in such scattering experiments the angular distribution is very insensitive to atomic numbers, particularly if the atomic numbers are very similar. To achieve a chemical distinction of the partial structure factors, it is necessary to use more elaborate methods such as anomalous SAXS [5] or SANS using different isotopes [6].

In an energy filtered transmission electron microscope (EFTEM) $[7,8,9]$, it is possible to select the energy of the transmitted electrons which are used for image recording. The elemental maps calculated from these images provide chemically resolved data.

These maps contain all information about the distribution of the elements in the specimen. It is therefore possible to extract partial structure factors from elemental maps. The procedure will be described below.

\section{Signal in an elemental map}

When adjusting the energy selecting aperture to the core loss region, the signal $I_{a}(\boldsymbol{\rho})$ of an elemental map can be 
approximated by a sum of single atom intensities $I_{s, a}(\boldsymbol{\rho}-$ $\boldsymbol{\rho}_{i}$ ) of element $a$ with $\boldsymbol{\rho}_{i}$ being the atom positions [10],

$$
I_{a}(\boldsymbol{\rho})=\sum_{i} I_{s, a}\left(\boldsymbol{\rho}-\boldsymbol{\rho}_{i}\right)
$$

The vector $\rho$ indicates the coordinates in the object plane. Introducing the projected atomic distribution function

$$
n_{a}(\boldsymbol{\rho})=\sum_{i} \delta\left(\boldsymbol{\rho}-\boldsymbol{\rho}_{i}\right)
$$

the signal in the elemental map can be rewritten as a convolution

$$
I_{a}(\boldsymbol{\rho})=\int I_{s, a}\left(\boldsymbol{\rho}-\boldsymbol{\rho}^{\prime}\right) n_{a}\left(\boldsymbol{\rho}^{\prime}\right) d^{2} \boldsymbol{\rho}^{\prime}
$$

Using the Fourier transform of the signal we arrive at

$$
\hat{I}_{a}(\boldsymbol{u})=\hat{I}_{s, a}(\boldsymbol{u}) \cdot \hat{n}_{a}(\boldsymbol{u}),
$$

with the Fourier transform of the single atom intensity

$$
\hat{I}_{s, a}(\boldsymbol{u})=\int I_{s, a}(\boldsymbol{\rho}) \exp (2 \pi i \boldsymbol{u} \boldsymbol{\rho}) d^{2} \boldsymbol{\rho},
$$

and of the projected atomic distribution

$$
\hat{n}_{a}(\boldsymbol{u})=\int n_{a}(\boldsymbol{\rho}) \exp (2 \pi i \boldsymbol{u} \boldsymbol{\rho}) d^{2} \boldsymbol{\rho} .
$$

Integrating an atomic intensity over the whole field of view yields the electron incident intensity $I_{0}$ multiplied by the scattering cross section $\sigma\left(E_{a}, \Delta E, \theta_{0}\right)$ depending on the energy loss $E_{a}$, the energy slit width $\Delta E$ and the objective aperture half angle $\theta_{0}$. Defining the inelastic transfer function as

$$
H_{a}(\boldsymbol{u})=\hat{I}_{s, a}(\boldsymbol{u}) /\left(I_{0} \cdot \sigma\left(E_{a}, \Delta E, \theta_{0}\right)\right),
$$

we arrive at a function describing the scattering process and the effect of the microscope on the imaging process. In the dipole approximation $H_{a}(\boldsymbol{u})$ depends on the element only via the energy loss. For the usual imaging parameters used in an EFTEM for the acquisition of elemental maps, e. g. small objective apertures to limit the scattering angle $[11,12]$, this approximation is very good. Therefore our Fourier transformed signal in the elemental map can be rewritten as

$$
\hat{I}_{a}(\boldsymbol{u})=I_{0} \cdot H_{a}(\boldsymbol{u}) \cdot \sigma\left(E_{a}, \Delta E, \theta_{0}\right) \cdot \hat{n}_{a}(\boldsymbol{u}) .
$$

For a well aligned stigmatic microscope $H_{a}(u)$ is rotationally symmetric. Additionally, for a randomly distributed, isotropic structure the average projected atomic distribution is also rotationally symmetric. Using a rotational average of the squared modulus of the signal in the elemental map, only the radially symmetric terms remain

$$
\left|\hat{I}_{a}(u)\right|^{2}=\left|I_{0} \cdot \sigma\left(E_{a}, \Delta E, \theta_{0}\right)\right|^{2} \cdot\left|H_{a}(u)\right|^{2} \cdot\left|\hat{n}_{a}(u)\right|^{2} .
$$

The squared modulus of the atomic distribution $\left|\hat{n}_{a}\right|^{2}$ is proportional to the partial structure factor $S_{a a}(u)$ of the element a. This structure factor is equivalent to the structure factor used for the description of scattering experiments as shown in (2). We obtain

$$
S_{a a}(u) \propto\left|\hat{I}_{a}(u)\right|^{2} /\left|H_{a}(u)\right|^{2} .
$$

Because of the chemical sensitivity of the elemental map, it is also possible to acquire the partial structure factor for other elements, $S_{b b}(u)$, as well as the combined partial structure factor

$$
S_{a b}(u) \propto \frac{\operatorname{Re}\left[\hat{I}_{a}(u) \cdot \hat{I}_{b}^{*}(u)\right]}{H_{a}(u) \cdot H_{b}(u)} \propto \operatorname{Re}\left[\hat{n}_{a}(u) \cdot \hat{n}_{b}^{*}(u)\right] .
$$

These formulas can easily be extended to more than two elements.

\section{Experimental procedures}

For our experiments we used an alloy of 45 at\% iron and 55 at\% chromium as a model system. This alloy shows spinodal decomposition $[13,14]$. We homogenized our specimen at $1100{ }^{\circ} \mathrm{C}$ to ensure a homogeneous distribution before tempering it at $550{ }^{\circ} \mathrm{C}$ for two weeks. The alloy blocks were cut into discs and electrolytically polished for use in the TEM.

We chose this specimen system because comparable structure factors were already determined. Previous works by other groups using other methods like SANS and SAXS provide data for comparisons $[15,16]$.

For our experiments we used a Zeiss Libra 200FE transmission electron microscope with an in-column $\Omega$-filter. It is equipped with a Gatan Ultrascan 4000 slow scan CCDcamera. When recording elemental maps it is usually used in the binning 2 mode to obtain sufficient intensity per pixel resulting in $2 \mathrm{k}$ by $2 \mathrm{k}$ image sizes.

To calculate an elemental map we use three windows before the characteristic edge to extrapolate the background intensity pixel by pixel. The background is then subtracted from the image of the post-edge window resulting in an elemental map. In figure 1 we illustrate the procedure.

In addition to the description of the scattering and the imaging process in the TEM with $H_{a}(u)$ the detection process still needs to be taken into account. A slow-scan CCD-camera suffers from a broadening of the incident signal resulting in a point spread function. The Fouriertransform of this point spread function is called the modulation transfer function $M(u)$. Essentially, higher spatial frequencies in the Fourier-transformed image are attenuated or equivalently in real space the contrast for small image details is reduced. $M(u)$ is either supplied by the camera manufacturer or can be measured using the sharp edge method described elsewhere $[17,18]$.

The specimen drift during image acquisition breaks the radial symmetry of the Fourier-transformed image. The effect of the drift results in a multiplicative cardinal sine $D(u)$ in the drift direction. It is possible to correct for this effect if the drift is sufficiently small and the zeros are not 
Energy loss in eV

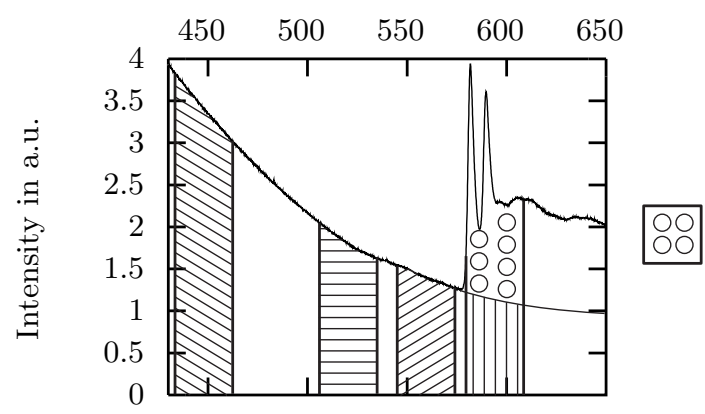

Energy selection for EFTEM images

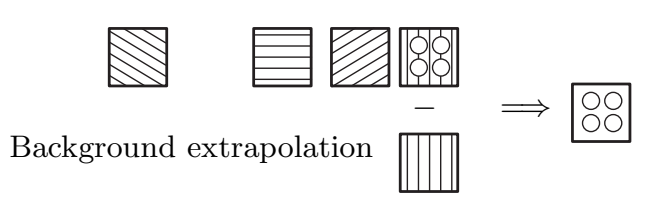

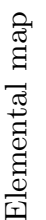

Figure 1. Procedure to determine elemental maps, e.g. for chromium. Using a an electron energy loss spectrum of the selected specimen position one selects the appropriate energy values and slit widths for the EFTEM images. The three preedge EFTEM images are used to extrapolate the background of the post-edge image. Subtracting the background from the post-edge image results in the elemental map.

in the region of interest.

As the electron detection process is a counting process one has also to consider the effect of the shot-noise of the detector. This effect can be described by the noise transfer function $N(u)$ in Fourier-space [18]. The experimentally recorded total Fourier-transformed image can therefore be written as $[12,19]$

$$
\begin{array}{r}
\left|\hat{I}_{a, \exp }(u)\right|^{2}=|N(u)|^{2}+|D(u)|^{2} \cdot|M(u)|^{2} \\
\cdot\left|H_{a}(u)\right|^{2} \cdot S_{a a}(u) \cdot \text { const. }
\end{array}
$$

In figure 2 we show the essential data needed to determine the structure factor for a single element. The elemental maps were recorded with three pre-edge and one post-edge image to allow for a better fit and less noise in the background subtraction [20]. In addition, a set of images without specimen were taken to calculate the noisetransfer-function. The elemental maps were then Fouriertransformed and circularly averaged followed by correction for $N, M$ and $H$. In most cases we could avoid the drift correction by using a new recording procedure. By dividing the illumination time of the camera from $300 \mathrm{~s}$ into $20 \cdot 15 \mathrm{~s}$ time slots, we could correct for image drift during acquisition time and use a larger image area [21]. The correction for $N(u)$ proved to be difficult. To determine $N(u)$, we had to use empty images taken at much higher intensities than the inelastically filtered images which resulted in differences depending on spatial frequency. The problem could be solved by adding a constant to $N(u)$. The value of the constant can be determined by comparing the gradients of $\left|\hat{I}_{a, \exp }(u)\right|^{2}$ and $|N(u)|^{2}$ in a region of

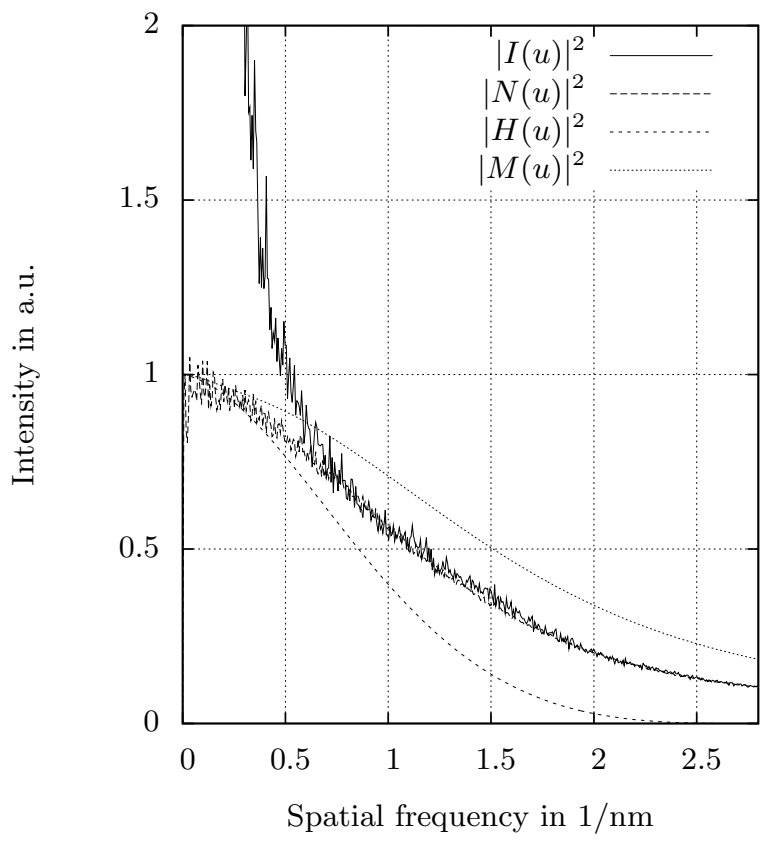

Figure 2. Rotationally averaged Fourier-transformed intensity $\left|\hat{I}_{a}(u)\right|^{2}$ of the elemental map for chromium and correctional factors for noise $|N(u)|^{2}$, scaled for correction, modulation transfer function $|M(u)|^{2}$ of the slow scan CCD-camera and the inelastic transfer function $\left|H_{a}(u)\right|^{2}$ describing the imaging process.

$u$ where $\left|H_{a}(u)\right|^{2}$ tends to zero.

The procedure is presented in figure 3 in form of a block diagram. It shows step by step how to implement the different corrections into the work flow.

The procedure to determine the mixed structure factor $S_{a b}(u)$ is quite similar. The Fourier transformed elemental map for element $a$ is multiplied by the complex conjugate of the Fourier transformed elemental map for element $b$. The real part of the resulting diffractogram is then corrected in the same fashion as before.

\section{Results}

The elemental maps necessary to calculate the structure factors were taken at a magnification of 195k. This assures a well resolved structure in the maps. An energy width of $33 \mathrm{eV}$ was chosen to allow for enough intensity without taking too much time in the recording process. Figure 4 shows the elemental map of chromium with the post-edge window being placed at $595 \mathrm{eV}$.

The elemental map for iron was taken with a post-edge window at $725 \mathrm{eV}$ with the same slit width as before. It is presented in figure 5. It is noisier than the chromium map due to the smaller inelastic scattering cross section at higher energy losses.

Looking at the disordered structure in the elemental maps 


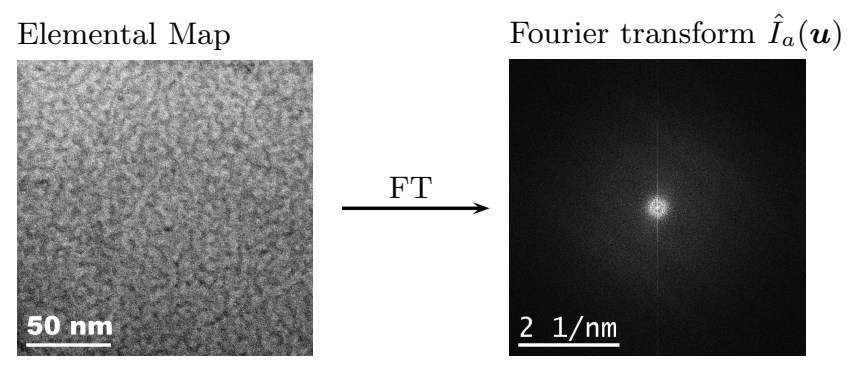

$\stackrel{\text { Squared modulus }}{\text { Circular average }}$

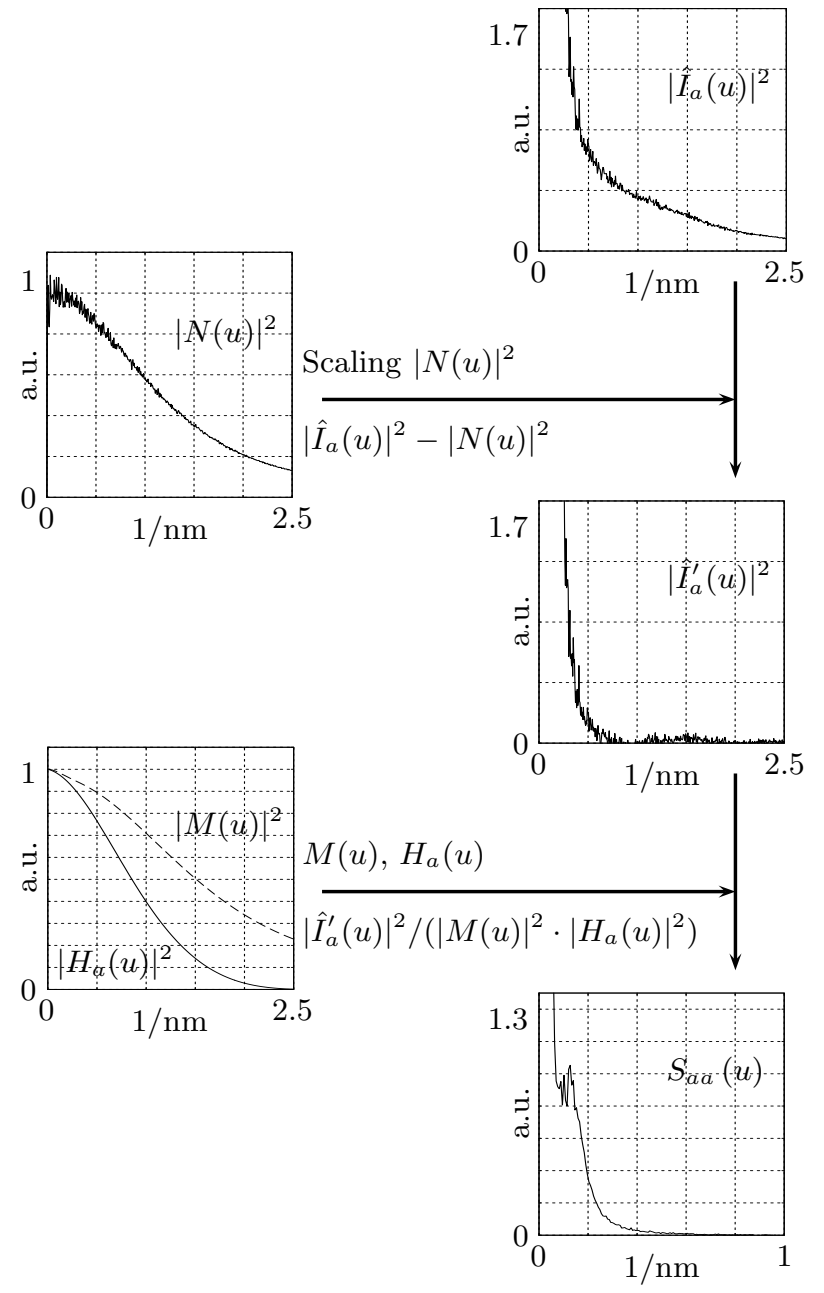

Figure 3. Data processing scheme for the determination of the partial structure factor for element $a$. The squared modulus of the Fourier transformed elemental map is circularly averaged. The resulting function $\left|\hat{I}_{a}(u)\right|^{2}$ is then corrected with the noise transfer function $N(u)$, the modulation transfer function $M(u)$ and the inelastic transfer function $H(u)$, yielding the partial structure factor for element $a$.

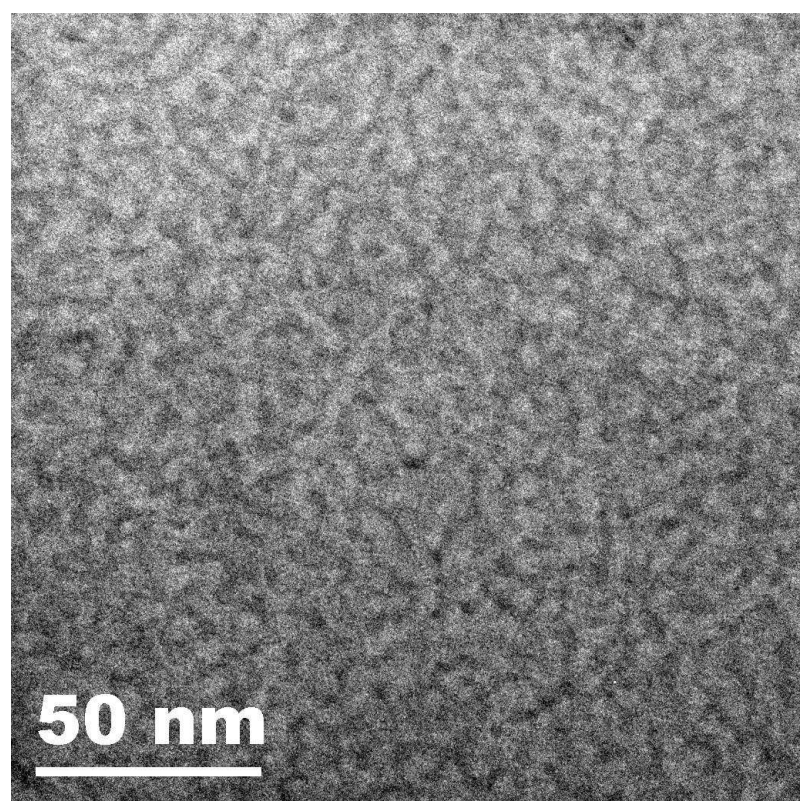

Figure 4. Elemental map of chromium, magnification 195k, pre-edge energies: $440 \mathrm{eV}, 525 \mathrm{eV}$ and $558 \mathrm{eV}$, post-edge energy: $595 \mathrm{eV}$, slit width $33 \mathrm{eV}$.

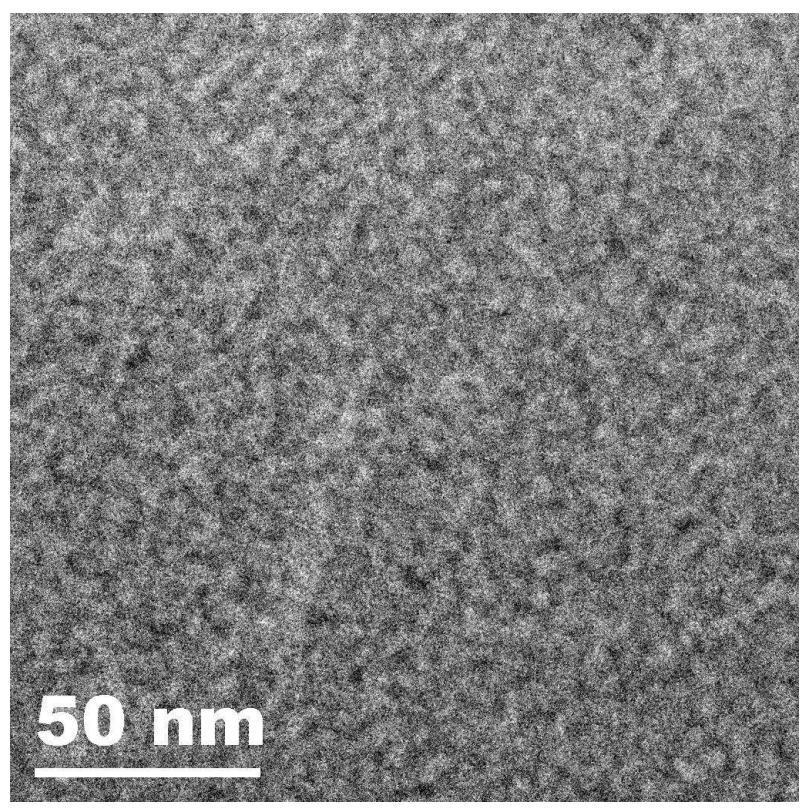

Figure 5. Elemental map of iron, magnification 195k, pre-edge energies: $624 \mathrm{eV}, 657 \mathrm{eV}$ and $690 \mathrm{eV}$, post-edge energy: $725 \mathrm{eV}$, slit width $33 \mathrm{eV}$.

it becomes obvious why the description of disordered structures via structure factors is necessary. It allows to determine the mean structure size in the specimen which in turn can be related to e.g. mechanical or magnetic properties of the material. The partial and mixed structure factors are shown in figure 6 . The similar behaviour of the partial and the mixed structure factors of iron and chromium is not surprising as the specimen consists of only two elements. They also show comparable behaviour to structure factors recorded with SANS by LaSalle et al. [15]. The difference 
in noise between the chromium and the iron map do not have a pronounced influence on the partial and the mixed structure factors, because the noise is strongly suppressed using the circular average.

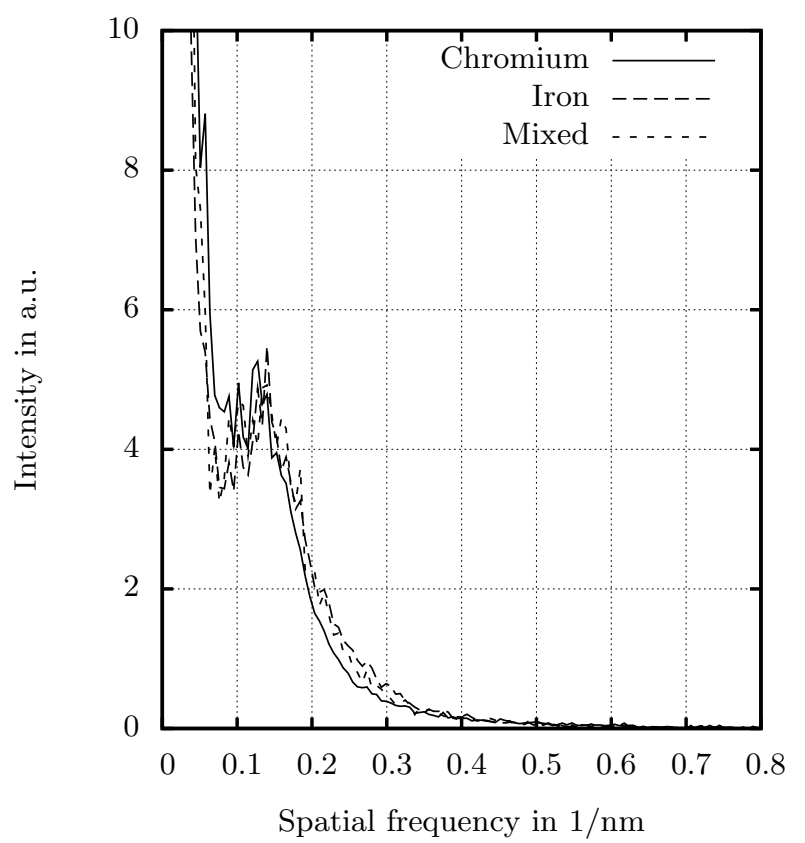

Figure 6. Partial and mixed structure factors of chromium and iron.

\section{Conclusions}

We have demonstrated that applying EFTEM to disordered structures results in well resolved structure factors. It is possible to acquire partial structure factors of a multi element specimen allowing for a chemical distinction. For TEM we need only very small specimens. The method presented here could therefore be a valuable alternative to small angle scattering, if the specimen size is not sufficient for other methods making it interesting e.g. for quality control. Another advantage is the high availability of TEMs equipped with imaging energy filters making this method easily accessible.

We wish to dedicate this article to Christian Colliex, who has introduced one of us (H.Kohl) into scanning transmission electron microscopy. Many inspiring discussions with him and his continuous support are gratefully acknowledged.

We would like to thank Prof. G. Schmitz and Dr. D. Baither from the Institut für Materialphysik for invaluable discussions and their support in specimen preparation.

Financial support of the Deutsche Forschungsgemeinschaft (DFG) under project Ko885/8-1 is gratefully acknowledged.

\section{References}

1. C. Colliex and B. Jouffrey, CR Acad. Sc. 270, (1970) 673

2. N.E. Cusack, The Physics of Structurally Disordered Matter (IOP Publishing, Philadelphia 1987)

3. A. Guinier, Small-angle Scattering of X-rays (Wiley, New York 1955)

4. L. Reimer, Transmission Electron Microscopy, Physics of Image Formation and Microanalysis, Springer Series in Optical Sciences 36 (Springer, Berlin 1997)

5. D.C. Bazin et al, J. Phys. Chem. B 101, (1997) 11040

6. M. Schaal et al, Z. Naturf. 43a, (1988) 1061

7. L. Reimer, Energy-Filtering Transmission Electron Microscopy, Springer Series in Optical Sciences 71 (Springer, Berlin 1995)

8. A. Berger and H. Kohl, Microscopy Microanalysis Microstructures 3, (1992) 159

9. R.F. Egerton, Electron Energy-Loss Spectroscopy in the Electron Microscope (Plenum Press, New York 1996)

10. H. Kohl and H. Rose, Adv. Electron. El. Phys. 65, (1985) 173

11. R. Knippelmeyer and H. Kohl, Journal of Microscopy 194, (1999) 30

12. R. Knippelmeyer, Ph.D. thesis, University of Münster, 2000

13. J.W. Cahn, Acta metall. 9, (1961) 795

14. J.S. Langer et al., Phys. Rev. A 11, (1975) 1417

15. J.C. LaSalle and L.H. Schwartz, Acta metall. 34, (1986) 989

16. M.K. Miller et al, Acta metall. 43, (1995) 3385

17. C. Hülk, Ph.D. thesis, University of Münster, 1998

18. R.R. Meyer et al, Ultramicroscopy 85, (2000) 9

19. R. Knippelmeyer, A. Thesing and H. Kohl, Zeitschrift für Metallkunde 94, (2003) 282

20. B. Gralla, diploma thesis, University of Münster, 2003.

21. T. Heil and H. Kohl, Ultramicroscopy 110, (2010) 745 